\title{
Características de un grupo de hombres presos por violencia de género y variables asociadas con la violencia contra las mujeres
}

\author{
Noelia Rodríguez-Espartal \\ Recibido: 30/10/2013 - Aceptado: 04/12/2013
}

\begin{abstract}
Resumen
La violencia de género es un grave problema social que afecta en la actualidad a un importante número de mujeres de diferentes culturas. Este trabajo se presenta como una nueva perspectiva en el abordaje de la violencia de género, pues se centra en ofrecer las características que presentan un grupo de hombres presos por violencia contra las mujeres. Se analizaron diferentes variables sociodemográficas, así como otras relacionadas con la violencia de género o protectoras frente a esta. Los resultados del trabajo indican que los maltratadores componen un grupo heterogéneo y que no todos se pueden englobar en las tipologías existentes, con lo que el trabajo encaminado a su reinserción y rehabilitación debe estar adaptado a estas características.
\end{abstract}

\section{Palabras clave}

Violencia de género, maltratadores, características, prisión.

\begin{abstract}
Gender violence is a serious social problem that currently affects a significant number of women from different cultures. This paper presents a new perspective in addressing gender-based violence, as it focuses on delivering the features that have a group of men imprisoned for violence against women. We analyzed different sociodemographic and other related domestic violence or protection against it. The results of the study indicate that batterers compose a heterogeneous group, and not all can be included in existing typologies, so that work towards their reintegration and rehabilitation should be tailored to these characteristics.
\end{abstract}

\section{Keywords}

Gender violence, batterers, characteristics, prison.

\section{Desarrollo}

La violencia de género es el resultado de una relación desigual con dos protagonistas. En los casos más habituales, la mujer es la víctima y el hombre es el victimario, y, aunque no debemos pasar por alto relaciones en las que esto ocurre a la inversa o en las que ambos miembros de la pareja son del mismo sexo, su abordaje no supone el objetivo del presente trabajo, sino que nos centraremos en la parte responsable de la acción violenta en el binomio hombre-mujer.

Hombre golpeador (Dutton, 1997), hombre abusador (Mullender, 2000), hombre maltratador, hombre agresor, hombre que incurre en malos tratos en su relación de pareja, hombre violento con su pareja, etc., son algunas de las denominaciones que se han dado a esta figura violenta. 
Consideramos necesario incluir al hombre en el análisis de la violencia de género, pues, además de ser parte importante en esta, el hecho de no incorporar el punto de vista masculino da como resultado una mirada parcial y una perspectiva incompleta respecto al problema (Duarte, Gómez y Carrillo, 2010). En el estudio de la violencia de género, tradicionalmente se ha prestado mayor atención al estudio de la mujer víctima, a sus características, su recuperación, las variables implicadas y explicativas de su situación, etc., pero muy pocas veces se lanzan cuestiones sobre el actor principal, sobre los hombres que ejercen esta violencia, sobre sus objetivos y sus motivaciones. Según Lorente (2007), "el hombre vuelve a ser el gran ausente en unas conductas protagonizadas por él, para así evitar su responsabilidad social, tanto en la construcción de las conductas como en la autoría de los casos" (pp. 26).

En este sentido, y hasta el momento, la mayoría de investigaciones se han centrado en establecer tipologías de hombres violentos contra su pareja heterosexual; y los resultados indican que no se trata de un grupo homogéneo (Cavanaugh y Gelles, 2005; Delsol, Margolin y John, 2003; Holtzworth-Munroe, 2000; Johnson y cols. 2006), al igual que ocurre con las mujeres maltratadas.

En el caso de los hombres, existen diferentes clasificaciones tipológicas de los maltratadores. Así, principalmente nos encontramos con las que se centran en las características psicopatológicas que presentan estos hombres y aquellas que se basan en variables de maltrato, psicológicas y fisiológicas (Amor y cols., 2009). No obstante, y aunque en estas tipologías se dan diferencias, existe un gran consenso respecto a que no todos los maltratadores son iguales (Amor, Echeburúa y Loinaz, 2009; Echeburúa, FernándezMontalvo y Amor, 2003; 2006; Fernández-Montalvo, Echeburúa y Amor, 2005). Por lo que, teniendo esto en cuenta, los programas de intervención no serán igualmente eficaces en todos los maltratadores; e incluso Babcock, Green y Robie (2004) han señalado su baja influencia para conseguir una reducción de la reincidencia en los comportamientos violentos.

En el amplio debate existente sobre tipologías de maltratadores, destacan dos grupos de investigadores; en concreto, el de Gottman y cols. (1995) y el de HoltzworthMunroe y Stuart (1994). Pasaremos a describir brevemente cada una de estas clasificaciones (para una revisión más amplia consultar las obras de los autores).

- Clasificación de Gottman y cols. (1995). Realizada en contexto de laboratorio, encontraron dos tipos de maltratadores en función de su respuesta cardíaca diferencial ante una discusión de pareja. Así, los autores hablan de maltratadores tipo 1 (cobra), con un tipo de violencia instrumental; y maltratadores tipo 2 (pitbull), en los que se observa una violencia impulsiva.

\section{Clasificación de Holtzworth-Munroe y Stuart (1994).}

Estos autores se centran, para realizar su clasificación de los maltratadores, en su funcionamiento psicológico, la extensión de la violencia y la gravedad de las conductas violentas. Así, establecen la existencia de tres tipos de maltratadores: limitados al ámbito familiar, borderline/ disfóricos y violentos en general/antisociales.

Ambas tipologías han sido replicadas empíricamente en estudios más recientes, habiéndose realizado además nuevas clasificaciones basadas en otras dimensiones de interés. De este modo, los estudios realizados proponen la existencia de dos, tres e incluso cuatro tipos de agresores (para una descripción más exhaustiva consultar Amor y cols., 2009).

La mayoría de los estudios sobre tipos de maltratadores identifican tres categorías: a) limitados al ámbito familiar, con riesgo de violencia bajo; b) borderline/disfóricos, con riesgo moderado de violencia; y c) violentos en general/ antisociales, con riesgo de violencia alto. Además, cada uno de los tipos de maltratadores se corresponde con diferentes niveles de extensión, frecuencia y gravedad de la violencia ejercida, así como de un determinado nivel de psicopatología.

En general, las tipologías resultan bastante estables con el paso del tiempo, sin darse evoluciones de uno a otro tipo (Holtzworth-Munroe y cols., 2003). Aunque, dependiendo del tipo de variables que se introduzcan en el estudio, irán surgiendo otras clasificaciones igualmente válidas e interesantes.

La utilidad de establecer una clasificación de maltratadores o de indicar sus principales rasgos es interesante desde muchos aspectos, pero, sobre todo, desde una perspectiva terapéutica y preventiva; de este modo se podrán seleccionar los tratamientos más adecuados a cada caso. Las tipologías no solo describen los tipos de maltratadores, sino que intentan abordar las causas y motivaciones que llevan a estos hombres a ser violentos con sus parejas (Babcock, Miller y Siard, 2003).

Es necesario seguir investigando para desarrollar un sistema clasificatorio de hombres violentos contra la pareja (Amor y cols., 2009); analizar la estructura de parejas violentas para llevar a cabo un tratamiento de 
ambos miembros (Cáceres y Cáceres, 2006; Dixon y Browne, 2003), así como considerar todos los factores implicados en la violencia de pareja (factores de riesgo de cada miembro, contextos e interacciones de la pareja, consecuencias en el entorno, etc.) (Amor y cols., 2009; Capaldi y Kim, 2007). Por ejemplo, en el caso de las víctimas que no desean separarse de sus maltratadores; en las parejas en las que ambos miembros son violentos entre sí, o cuando existe un desplazamiento de la violencia hacia los hijos (Amor y cols., 2009).

A pesar de toda la investigación al respecto - como ya hemos mencionado con anterioridad-, no se puede hablar de que exista un perfil único y específico de los agresores y sí algunas características que comparten algunos de ellos. Lo que no podemos olvidar es que los maltratadores constituyen un grupo heterogéneo, lo que debe ser tenido en cuenta a la hora de abordar su tratamiento. Existen muchas variables que se han asociado tradicionalmente a la violencia de género y que, en ocasiones, han servido para minimizarla o justificarla. A continuación vamos a pasar a considerar las más frecuentes.

Entre las características que presentan los hombres violentos con su pareja, cabe destacar (OMS, 2003; Organización Panamericana de la Salud, 2002): escaso control del comportamiento con la creencia de tener el derecho y el deber de hacer uso de la violencia como mecanismo para evitar la pérdida de poder y control, baja autoestima, trastornos de la personalidad y la conducta, falta de lazos emocionales y de apoyo, contacto temprano con la violencia en el hogar como víctimas directas o como testigos, historias familiares o personales marcadas por divorcios o separaciones. Este es otro tema controvertido cuando se habla de la violencia de género. Respecto a si los hombres violentos con su pareja han aprendido estas conductas en sus familias, existen casos en los que un porcentaje muy bajo de los maltratadores adultos ha sufrido en su niñez los efectos de la violencia de género en las relaciones de pareja (Cabrera, 2010). Sin embargo, si se incluye la perspectiva de género, que considera una definición más amplia de este tipo de violencia, nos encontramos con hombres que han tenido una infancia dura, donde el maltrato (entendido de forma más amplia que la mera violencia física) ha sido la norma y no la excepción. Son personas en las que se han depositado fuertes expectativas de ascenso en el futuro, con exigencias que han podido superar sus propias posibilidades y que han vivido en familias en donde el aspecto material se ha impuesto al emocional (Cabrera, 2010).

Son hombres socializados en la cultura patriarcal en lo que a las relaciones de pareja se refiere, por lo que se consideran dominantes respecto al sexo femenino, tanto en lo físico como en lo cognitivo. La socialización en estos contextos patriarcales lleva a considerar al hombre que posee la autoridad moral para utilizar la agresión hacia su pareja como modo de control y a que la mujer, también socializada en estos ideales, encuentre natural el control, la humillación e incluso en ocasiones el castigo físico (Cabrera, 2010).

Además, suele ser habitual la presencia de distorsiones cognitivas (relacionadas, sobre todo, con creencias equivocadas sobre los papeles sexuales y la inferioridad de la mujer y con la legitimidad de la violencia como forma adecuada de resolver conflictos); dificultades para controlar la ira (expresada en ocasiones con amenazas o agresiones físicas hacia la mujer o hacia algún objeto 0 persona querido por ella [Straus, 1993]); bajos grados de empatía, déficit en habilidades de comunicación y solución de problemas; alejamiento del hogar durante un tiempo indefinido, intentando restablecer posteriormente la comunicación con las relaciones sexuales (Dobash 1979; Dutton y Golant, 1997; Echeburúa y Corral, 1998; Echeburúa y Amor, 2010; Lorente y Lorente, 1998; Lorente 2011; Norlander y Eckhardt, 2005).

Suelen emplear diferentes estrategias de afrontamiento para eludir la responsabilidad de sus conductas violentas (entre ellas, justificar, minimizar o negar la violencia ejercida) (Echeburúa y Corral, 1998).

Los maltratadores son además poco realistas, con expectativas muy elevadas sin considerar sus capacidades y destrezas. Piensan que son jefes con el derecho de actuar violentamente. Cosifican a la mujer, negándole su derecho a ser persona. Buscan las creencias religiosas que colocan a la mujer en una posición inferior y tienden a presumir de la inferioridad de las mujeres (Walker, 1979).

Algunas conductas comunes a estos tipos de hombres son: actuar impulsivamente, cambiar de trabajo, amigos, casa; vivir aislado de los demás; ser excesivamente posesivos y dependientes de la pareja; expresar la mayoría de las emociones como explosiones de ira debido a la gran dificultad que les supone la expresión emocional (Echeburúa y Amor, 2010; Norlander y Eckhardt, 2005); controlar y dominar a otros (Straus y cols., 1980; Walker, 1979); tomar decisiones de manera unilateral (Walker, 1984); abusar de drogas y alcohol (Gelles, 1974; Straus, Gelles y Steinmetz, 1981); incurrir en conductas de maltrato a los niños (Straus y cols., 1981); ser hostil, dominante.

Presentan síntomas psicopatológicos variados, como celos, ansiedad y estrés, abuso del alcohol, irritabilidad, etc., que 
pueden precipitar los episodios violentos (Echeburúa y Corral, 1998).

Los déficits psicológicos son totalmente compatibles con la imputabilidad del agresor. El maltratador puede ser penalmente responsable de su conducta, lo que no quiere decir que se le niegue el tratamiento, bien en régimen comunitario, bien en prisión. El objetivo principal y último de tratar a los hombres violentos en las relaciones de pareja es evitar las reincidencias para proteger a las futuras víctimas (Echeburúa y Amor, 2010).

Como hemos señalado anteriormente, el hombre está ocupando una posición de poder, y, generalmente, aquellos que están en situación de poder no quieren renunciar a él, por lo que no les interesa resolver los problemas de forma consensuada y recurren a la imposición para obtener beneficios particulares, algo que, si además está legitimado por el componente cultural, no solo parece natural sino que además resulta invisible (Connell, 1995; Hilberman, 1980).

Antes de concluir este apartado, nos gustaría reflexionar sobre las creencias que existen en la sociedad respecto al hombre maltratador; comprobar si existe coincidencia entre las características que presentan estos hombres y cómo se los ve en la calle. En este sentido, Duarte y cols. (2010) encuentran en un estudio que hombres y mujeres perciben al hombre maltratador como una persona que ejerce violencia porque tienen un pobre control de impulsos y un abaja autoestima, esto es, ejerce violencia porque no puede controlarse, no porque no quiera hacerlo. Estos resultados son alarmantes, pues corroboran la creencia de que la violencia que ejerce un hombre maltratador está vista como un componente interno y natural. Desde este punto de vista, el tratamiento con los maltratadores no sería posible, pues algo innato no es susceptible de ser modificado. No obstante, hay diferencias entre hombres y mujeres al respecto. Así, las mujeres creen que los hombres son violentos sin necesidad de aparentar socialmente y que dicha violencia no se debe a elementos externos como el alcohol, las drogas o la experiencia previa con la violencia. Ellas relacionan más la violencia con aspectos intrínsecos, des-responsabilizando al hombre de cualquier efecto que el ejercicio de la violencia pueda tener. Por su parte, los hombres ven a los maltratadores con dificultades para controlar sus emociones que estas se ven aumentadas por el consumo de alguna droga. En este sentido, el hombre maltratador no lo es por naturaleza, sino porque existen factores extrínsecos que lo hacen ser violento. Además, tanto hombres como mujeres consideran que hay un cierto tipo de violencia legítima, e incluso deseable, que implica una connotación disciplinaria y que son acciones que son percibidas como justas por la sociedad donde se ejerce (Duarte y cols., 2010). En otras ocasiones, algunas manifestaciones de violencia se producen como formas de ser, apelando a la esencia natural de la persona, viéndose incluso como una forma de expresión afectiva por parte del hombre hacia la mujer, idea que es compartida por hombres y mujeres (Ramírez y Vargas, 1998).

Además, tanto mujeres como hombres parecen estar de acuerdo en que lo emocional no es terreno de lo masculino (Duarte y cols., 2010). Las mujeres manifiestan que socialmente se tolera la violencia del hombre, mientras que los hombres relacionan más la masculinidad con el ejercicio de la violencia; sus creencias tienden a vincular el ser violentos con factores extrínsecos. El peligro que entraña esta postura - como hemos comentado anteriormentees que ni hombres ni mujeres responsabilizan al hombre que es violento con su pareja de sus actos, sino que lo justifican como parte del papel social que le toca representar en la sociedad. En este sentido, se podría decir que el género estructura los discursos de tal manera que se mantiene el statu quo de la desigualdad.

De este modo, podemos concluir que resulta necesario e imprescindible conocer cómo son los hombres que ejercen la violencia contra las mujeres para, de este modo, adaptar las intervenciones a sus necesidades, con el objetivo último de proteger a las víctimas de esta violencia. En este sentido, nos planteamos el objetivo de identificar las características de un grupo de hombres maltratadores con la importante innovación de estudiar a aquellos que se encuentran en prisión por este delito.

\section{Método}

\section{Participantes y procedimiento}

En el presente estudio participaron un total de 97 hombres presos por delitos relacionados con la violencia de género y que cumplen su pena en la Institución Penitenciaria Provincial de Jaén, Jaén II.

Los participantes completaron los cuestionarios dentro de la institución penitenciaria. Además, se recogió una serie de datos sociodemográficos con el objetivo de ofrecer la mayor cantidad de información sobre ellos.

En cuanto a las variables sociodemográficas, tenemos un grupo de hombres que se encuentran entre los 22 y los 69 años ( $M=39.75, s d=11.10)$, y con una condena que va desde los 3 años y medio hasta los 20 años ( $M=4, s d=3.01$ ). En cuanto al nivel de estudios, la situación laboral, el nivel 
socioeconómico y el estado civil, pueden verse los datos en las figuras 1 a 4. (Insertar gráficas correspondientes.)

\section{Instrumentos}

Para la evaluación de los participantes, se elaboró un cuestionario que contenía, entre otras, las siguientes pruebas:

- Variables sociodemográficas: edad, nacionalidad, nivel de estudios, trabaja, estado civil, duración de la condena.

- Inventario de Personalidad NEO-FFI (Costa y McCrae, 1999; versión española de Cordero y cols., 1999).

- Inventario de Pensamientos Distorsionados sobre la mujer y sobre el uso de la violencia (IPDVM) (Echeburúa y Fernández-Montalvo, 1998).

- Cuestionario de Agresividad CDA (Buss y Perry, 1999; versión española de Andreu y cols., 2002).

- Cuestionario de Ansiedad STAXI-2 (SPielberger, 1988; versión española de Miguel-Tobal y cols., 2001).

- Cuestionario de Inteligencia Emocional TMMS-24 (Fernández-Berrocal y cols., 2004).

- Cuestionario de Inteligencia Emocional SSRI (Schutte y cols., 1998; versión española de Chico, 1999).

- Cuestionario de Alexitimia TAS-20 (Bagby y cols., 1994; versión española de Martínez-Sánchez, 1996).

- Escala de Autoestima de Rosenberg (1965; versión española de Fernández-Montalvo y Echeburúa, 1997).

- Cuestionario de Impulsividad Bis-11 (Barrat y cols., 1995; adaptación española de Oquendo y cols., 2001).

- Escala de Rol de género (adaptación de Cejka y Eagly, 1999).

- Escala de Cultura del Honor (López-Zafra, 2007).

- Cuestionario de Tácticas de control y celos (Instituciones Penitenciarias, 2000).

- Escala de Apoyo social (AS-25 (Vaux y cols., 1986).

- Escala de Deseabilidad Social de Marlownw y Crowne (1960) (adaptación española de Ferrando y Chico, 2000).

\section{Resultados}

A continuación pasamos a describir los resultados obtenidos tras realizar las correspondientes Anovas, de modo que podamos ofrecer las características que presenta un grupo de hombres presos por delitos de violencia de género.
Para facilitar la comprensión de los resultados, estos se ofrecerán por variables.

En las dimensiones de personalidad, tenemos hombres con puntuaciones medio-altas en neuroticismo $(M=69.86$, $\mathrm{sd}$ $=28.34)$; bajas en extraversión $(M=35.44$, $s d=24.64) \mathrm{y}$ apertura $(M=36.72, \mathrm{sd}=24.75)$; y medias en amabilidad $(M=54.64, \mathrm{sd}=29.48)$ y responsabilidad $(M=56.64, \mathrm{sd}=$ 32.44).

Son hombres con altas puntuaciones, tanto en pensamientos distorsionados sobre la mujer $(M=8.00, \mathrm{sd}=1.88)$ como sobre el uso de la violencia $(M=8.79$, $s d=1.67)$; y con altas expectativas de cambio $(M=, \mathrm{sd})$.

En cuanto a agresividad, sus puntuaciones en las diferentes dimensiones son: agresividad física $(M=10.58, \mathrm{sd}=3.41)$, agresividad verbal $(M=18.67, \mathrm{sd}=4.31)$, ira con resentimiento $(M=22.53, \mathrm{sd}=7.39)$ y hostilidad o sospecha $(M=9.06, s d=3.33)$, presentando bajos niveles en todas ellas.

Son hombres que presentan puntuaciones bajas en las dimensiones de la ira: ira rasgo total $(M=36.44, \mathrm{sd}=26.28)$, temperamento $(M=27.33$, $\mathrm{sd}=22.91)$, reacción $(M=56.31$, $\mathrm{sd}=29.99)$, expresión de la ira total $(M=38.86, \mathrm{sd}=28.91)$, ira externa $(M=33.00, \mathrm{sd}=25.24)$, ira interna $(M=35.72$, $\mathrm{sd}$ $=27.61)$, control de la ira $(M=20.11, s d=5.87)$ e ira estado total $(M=52.36, s d=17.17)$.

Presentan una baja inteligencia emocional en todos los componentes, salvo en percepción emocional: percepción emocional $(M=29.67, \mathrm{sd}=6.92)$, comprensión emocional $(M$ $=28.97, \mathrm{sd}=7.21)$, regulación emocional $(M=21.50, \mathrm{sd}=$ 4.22), inteligencia emocional total $(M=3.66, s d=.72)$ y una alta autoestima $(M=, \mathrm{sd})$. Datos que se ven apoyados por las altas puntuaciones que presentan en alexitimia: total $(M=$ $55.89, s d=12.31)$, dificultad para la discriminación de señales emocionales $(M=19.08, \mathrm{sd}=7.54)$, dificultad en la expresión verbal de emociones $(M=15.11, \mathrm{sd}=4.44)$ y pensamiento orientado hacia detalles externos $(M=21.78, s d=4.41)$.

Por su parte, en cuanto a la impulsividad, presentan puntuaciones bajas en todas las dimensiones: impulsividad total $(M=42.86, \mathrm{sd}=12.03)$, impulsividad cognitiva $(M=$ 13.22, $\mathrm{sd}=3.81)$, impulsividad motora $(M=12.72, \mathrm{sd}=6.44)$ e improvisación y ausencia de planificación ( $M=15.53$, sd $=6.25$ ).

En cuanto al papel de género, son hombres que se identifican más con el papel de género femenino $(M=14.31, \mathrm{sd}=6.00)$ que con el masculino $(M=10.31$, $\mathrm{sd}=3.88$ ). 
Sus puntuaciones en cultura del honor indican que estamos ante un grupo de hombres que concede alta importancia a este aspecto en sus relaciones, presentando valores medio-altos en todas las dimensiones de la escala: cultura del honor total $(M=3.51$, $\mathrm{sd}=.25)$, honor individual $(M=3.7, \mathrm{sd}=.47)$, sociedad y leyes en torno al honor ( $M=3.8$, sd $=.47$ ) y legitimidad en el uso de la violencia ante una ofensa $(M=3.3, \mathrm{sd}=.36)$.

Respecto a los celos, piensan que son ejercidos en mayor medida por la mujer ( $M=23.47$, $s d=1.34$ ) que por el hombre $(M=16.89, \mathrm{sd}=7.23)$.

Por último, presentan altas puntuaciones en apoyo social $(M=76.86, \mathrm{sd}=9.32)$ y bajas en deseabilidad social $(M=19.00, \mathrm{sd}=4.26)$.

\section{Conclusiones}

El presente trabajo supone un acercamiento al estudio de las características que presentan los hombres maltratadores que se encuentran en prisión cumpliendo condena por delitos relacionados con la violencia de género.

A partir de los resultados obtenidos en el presente trabajo, podemos concluir que el hombre que ejerce violencia contra las mujeres presenta un amplio abanico de características y que el maltratador puede ser cualquiera.

Las características obtenidas en este grupo de hombres no coinciden con las de las tipologías previas propuestas por diferentes autores (Gottman y cols., 1995; HoltzworthMunroe y Stuart, 1994), lo que refuerza aún más la idea de que el maltratador presenta una amplia heterogeneidad de características.

En este sentido, la utilidad de realizar este tipo de investigaciones, más que establecer tipologías de hombres maltratadores, persigue un objetivo terapéutico y preventivo, con el fin de seleccionar los tratamientos de intervención más adecuados y que permitan su mejor aprovechamiento y desarrollo en la reducción de las conductas, pensamientos y emociones relacionados con la violencia contra las mujeres. Además, el establecer tipologías persigue abordar las causas y motivaciones que llevan a estos hombres a ser violentos con sus parejas (Babcock, Miller y Siard, 2003). Además de considerar todos los factores implicados en la violencia de pareja (factores de riesgo de cada miembro, contextos e interacciones de la pareja, consecuencias en el entorno, etc.) (Amor, Echeburúa y Loinaz, 2009; Capaldi y Kim, 2007).

Es necesario implicar al hombre, actor de esta violencia, en su solución, pues, de lo contrario, la estamos convirtiendo en algo natural e incuestionable en las sociedades actuales. Resulta indispensable que los hombres se involucren en el desarrollo de las nuevas masculinidades, teniendo como herramienta de análisis la perspectiva de género, que resulta clave para la comprensión de las relaciones entre hombres y mujeres al visibilizar teórica y empíricamente la violencia del hombre contra la mujer (Comisión Europea, 2000; Ferreira, 1992; Osborne, 2001; Walker, 1984), y cuestionar el poder que les ha concedido la sociedad patriarcal.

Es necesario que el trabajo que se realice en este sentido no exalte las diferencias entre los géneros, sino que muestre que hombres y mujeres no son entes aislados, sino en mutua interrelación. Es necesario generar discursos alternativos de masculinidad-feminidad, para que la hegemonía no se convierta en una cárcel que impida a hombres y mujeres vivir en libertad, plenitud y con un verdadero acceso a una vida libre de violencia (Duarte y cols., 2010).

En conclusión, no hay que exaltar las diferencias entre los géneros, sino mostrar que hombres y mujeres no son entes aislados, sino en mutua interrelación. Sólo de esta forma caminaremos hacia una sociedad igualitaria, en la que no se exalten las diferencias de género y en la que la violencia contra las mujeres no tenga cabida.

\section{Limitaciones y líneas de futuro}

A pesar de que el presente trabajo supone un importante avance en el estudio del hombre maltratador, al abordar a aquellos que se encuentran en prisión no deja de tener ciertas limitaciones. En primer lugar, es necesario conocer la interrelación existente entre las diferentes variables para ver qué componentes tienen un mayor peso en el problema social de la violencia de género. Esto nos acercaría a conocer cada caso de violencia de género como un problema social específico y comprender qué rasgos son más relevantes y cómo se estructuran.

Además, es imprescindible agrupar a los hombres por las características que presentan para seleccionar el tratamiento más adecuado, que consiga los mejores resultados y que logre una generalización de las conductas al entorno del hombre. La prisión tiene una función de rehabilitación y reinserción social que debe cumplirse con este tipo de intervenciones. 


\section{APÉNDICE}
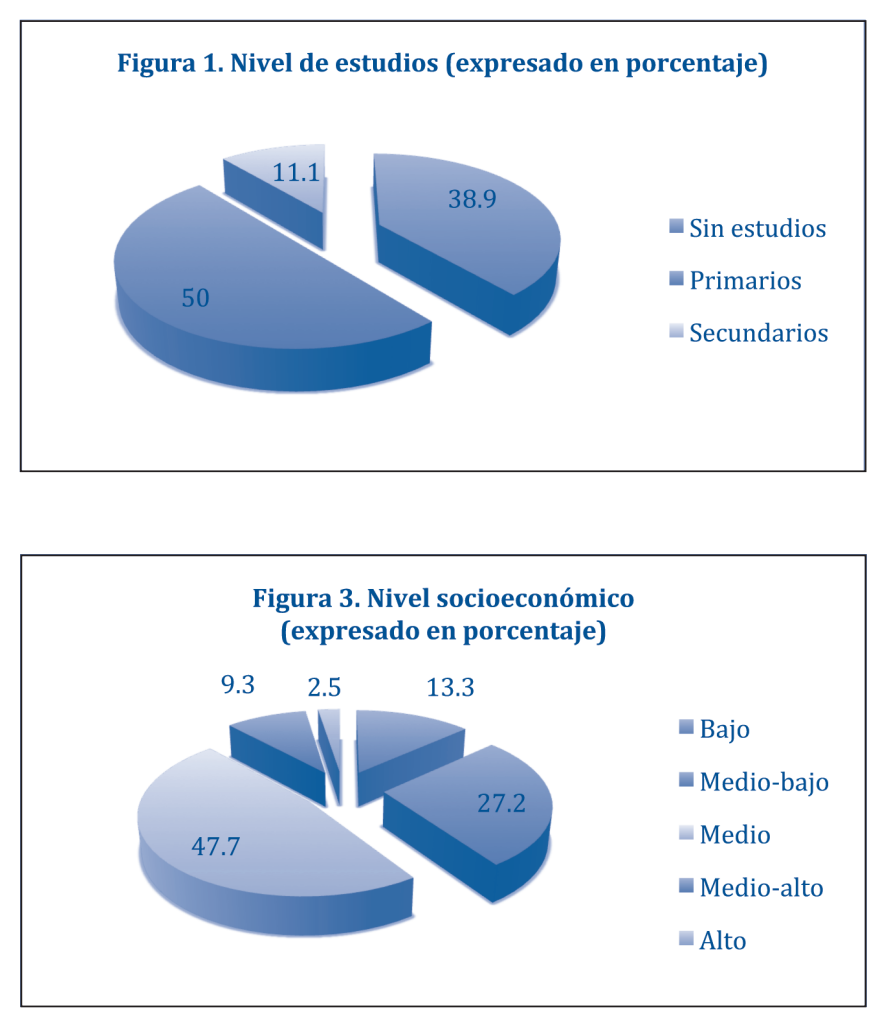

\section{Referencias}

Amor, P.J.; Echeburúa, E. y Loinaz, I. (2009). "¿Se puede establecer una clasificación tipológica de los hombres violentos con su pareja?". International Journal of Clinical and Health Psychology, 9 (3), 519-539.

Babcock, J.C.; Green, C.E. y Robie, C. (2004). "Does batterer's treatment work? A meta-analytic review of domestic violence treatment". Clinical Psychology Review, 23, 1023-1053.

Babcock, J.C.; Miller, S.A. y Siard, C. (2003). "Toward a typology of abusive women: Differences between partner-only and generally violent women in the use of violence". Psychology of Women Quarterly, 27, 153-161.

Cabrera, M. (2010). “Acercándonos al hombre que ejerce la violencia de género: clasificación y descripción de un grupo de maltratadores". Nómadas. Revista Crítica de Ciencias Sociales y Jurídicas, 25, 1-24.

Cáceres, A. y Cáceres, J. (2006). “Violencia en relaciones íntimas en dos etapas evolutivas". International Journal of Clinical and Health Psychology, 6, 271-284.

Capalid, D.M. y Kim, H.K. (2007). "Typological approaches to
Figura 2. Situación laboral (expresada en porcentaje)
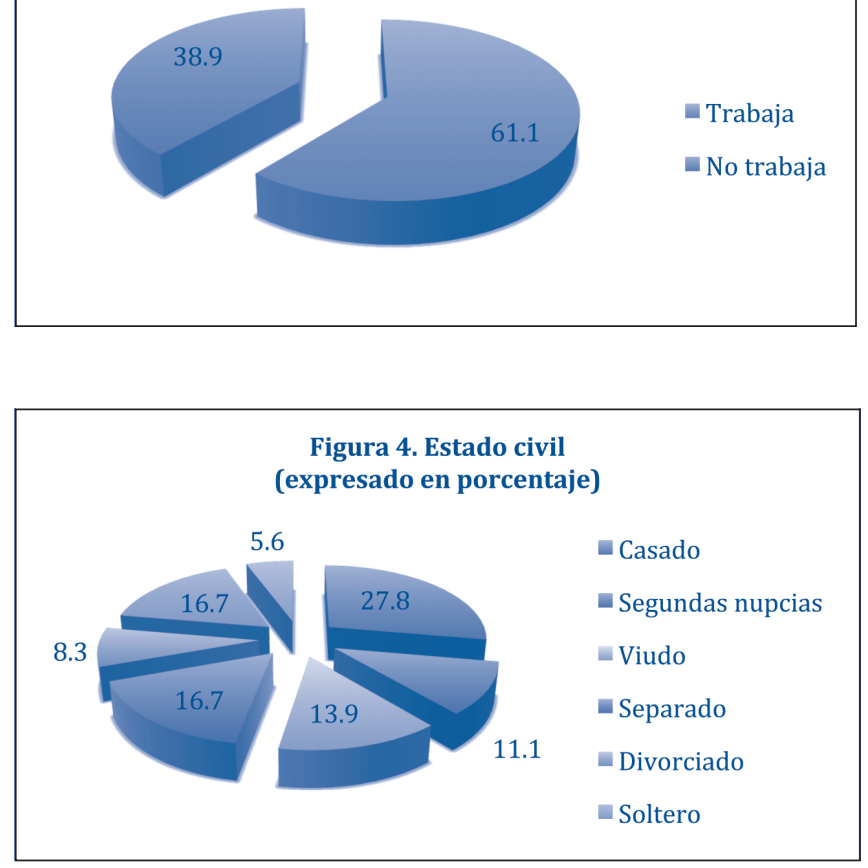

violence in couples: A critique alternative conceptual approach". Clinical Psychology Review, 27, 253-265.

Cavanaugh, M.M. y Gelles, R.J. (2005). "The utility of male domestic violence ofender typologies". Journal of Interpersonal Violence, 20, 155-166.

Comisión Europea (2000). Romper el silencio. Campaña europea contra la violencia doméstica. Luxembourg: Oficina de Publicaciones Oficiales de las Comunidades Europeas.

Connell, R.W. (1995). Masculinities: Knowledge, power, and social change. Cambridge: Polity Press.

Delsol, C.; Margolin, G. y John, R.S. (2003). "A typology of martially violent men and correlates of violence in a community sample". Journal of Marriage and Family, 54, 635-651.

Dixon, L. y Browne, K. (2003). "The heterogeneity of spouse abuse: A review". Aggression and Violent Behavior, 8, 107-130.

Dobash, R. (1979). Violence against Wives: A Case against the Patriarchy. New York: Free Press.

Duarte, E.; Gómez, J.F. y Carrillo, C.D. (2010). "Masculinidad y hombre maltratador: ¿pueden las creencias de 
hombres y mujeres propiciar la violencia de género?". Revista de Psicología, 19 (2), 7-30.

Dutton, D.G. y Golant, S.K. (1997). El golpeador. Un perfil psicológico. Buenos Aires: Paidós.

Echeburúa E. y Amor, P.J. (2010). "Perfil psicopatológico e intervención terapéutica con los agresores contra la pareja". Revista Española de Medicina Legal, 36 (3), 117-121.

Echeburúa, E. y Corral, P. (1998). Manual de violencia familiar. Madrid: Siglo XXI.

Echeburúa, E.; Fernández-Montalvo, J. y Amor, P.J. (2003). "Psychopathological profile of men convicted of gender violence: A study in the prisons of Spain". Journal of Interpersonal Violence, 18, 798-812.

Echeburúa, E.; Fernández-Montalvo, J. y Amor, P.J. (2006). "Psychological treatment of men convicted of gender violence: A pilot-Study in the Spanish prisons". International Journal of Offender Therapy and Comparative Criminology, 50, 57-70.

Fernández-Montalvo, J.; Echeburúa, E. y Amor, P.J. (2005). "Aggressors against women in prison and in community: An exploratory study of a differential profile". International Journal of Offender Therapy and Comparative Criminology, 49, 158-167.

Ferreira, G. B. (1992). Hombres violentos, mujeres maltratadas. Aportes a la investigación y tratamiento de un problema social. Buenos Aires: Sudamericana.

Gottman, J.M.; Jacobson, N.S.; Rushe, R.H.; Shortt, J.W.; Babcock, J.; La Taillade, J.J. y Waltz, J. (1995). "The relationship between heart rate activity, emotionally aggressive behavior, and general violence in batterers". Journal of Family Psychology, 9, 227-248.

Hilberman, E. (1980). "Overview: The 'Wife-beater's wife' reconsidered". American Journal of Psychiatry, 137, 1336-1347.

Holtzworth-Munroe, A. (2000). "A typology of men who are violent toward their female partners: Making sense of the heterogeneity in husband violence". Current Directions in Psychological Science, 9, 140-143.

Holtzworth-Munroe, A. y Stuart, G.L. (1994). "Typologies of male batterers: Three subtypes and the differences among them". Psychological Bulletin, 116(3), 476-497. Holtzworth-Munroe, A.; Meehan, J.C.; Herron, K.; Rehman, U. y Stuart, G.L. (2003). "Do subtypes of maritally violent men continue to differ over time?", Journal of consulting and Clinical Psychology, 71, 728-740.

Johnson, R.; Gilchrist, E.; Beech, A.R.; Weston, S.; Takriti, R. y Freeman, R. (2006). "A psychometric typology of U.K. domestic violence offenders". Journal of Interpersonal Violence, 21, 1270-1285.

Lorente, M. (2007). Violencia de género, educación y socialización: acciones y reacciones. Revista de Educación, 342, 19-35.

Lorente, M. (2011). "Igualdad y conciliación de la vida laboral, familiar y personal". Intervención en la Universidad de Jaén. Disponible en: http://diariodigital.ujaen.es/ node/26554

Lorente, M. y Lorente, J.A. (1998). Agresión a la mujer: maltrato, violación y acoso. Granada: Comares.

Mullender, A. (2000). La violencia doméstica. Una nueva visión de un viejo problema. Barcelona: Paidós.

Organización Mundial de la Salud (2003). "Informe mundial sobre la violencia y la salud. Organización Mundial de la salud", Washington.

Organización Panamericana de la Salud (2002). "Informe Mundial sobre la Violencia y la Salud". Washington, D.C.: OPS. En http://www.paho.org/Spanish/AM/PUB/ Violencia_2003.htm Consultado el 17 de octubre de 2013.

Osborne, R. (2001). (Ed.). La violencia contra las mujeres. Realidad social y políticas públicas. Madrid: Uned.

Straus, M.A. (1993). "Physical assault by wives: A major social problem". En: R.J. Gelles and D. Loseke (Eds.), Current controversies on family violence (pp. 67-87). Newbury Park, CA: Sage.

Straus, M.A.; Gelles, R.J. y Steinmetz, S.K. (1981). Behind closed doors: Violence in the American family. Anchor Books Edition.

Walker, L.E. (1979). The battered woman, New York: Harper and Row.

Walker, L.E. (1984). The battered woman syndrome, Nueva York: Springer. 